\title{
Ray representation of sound scattering by weakly scattering deformed fluid cylinders: Simple physics and application to zooplankton
}

Timothy K. Stanton

Department of Applied Ocean Physics and Engineering, Woods Hole Oceanographic Institution, Woods Hole, Massachusetts 02543

Clarence S. Clay

Department of Geology and Geophysics, University of Wisconsin, Madison, Wisconsin 53706

Dezhang Chu

Department of Applied Ocean Physics and Engineering, Woods Hole Oceanographic Institution, Woods Hole, Massachusetts 02543

(Received 28 April 1992; accepted for publication 2 July 1993)

\begin{abstract}
Data indicate that certain important types of marine organisms behave acoustically like weakly scattering fluid bodies (i.e., their material properties appear fluidlike and similar to those of the surrounding fluid medium). Use of this boundary condition, along with certain assumptions, allows reduction of what is a very complex scattering problem to a relatively simple, approximate ray-based solution. Because of the diversity of this problem, the formulation is presented in two articles: this first one in which the basic physics of the scattering process is described where the incident sound wave is nearly normally incident upon a single target (i.e., the region in which the scattering amplitude is typically at or near a maximum value for the individual) and the second one [Stanton et al., J. Acoust. Soc. Am. 94, 3463-3472 (1993)] where the formulation is heuristically extended to all angles of incidence and then statistically averaged over a range of angles and target sizes to produce a collective echo involving an aggregation of randomly oriented different sized scatterers. In this article, a simple ray model is employed in the deformed cylinder formulation [Stanton, J. Acoust. Soc. Am. 86, 691-705 (1989)] to describe the scattering by finite length deformed fluid bodies in the general shape of elongated organisms. The work involves single realizations of the length and angle of orientation. Straight and bent finite cylinders and prolate spheroids are treated in separate examples. There is reasonable qualitative comparison between the structure of the data collected by Chu et al. [ICES J. Mar. Sci. 49, 97-106 (1992)] involving two decapod shrimp and this single-target normal-incidence theory. This analysis forms the basis for successful comparison (presented in the companion article) between the extended formulation that is averaged over an ensemble of realizations of length and angle of orientation and scattering data involving aggregations of up to 100 's of animals.
\end{abstract}

PACS numbers: 43.20.Fn, 43.30.Gv, 43.30.Xm

\section{LIST OF SYMBOLS}

$p_{\text {scat }} \quad$ scattered pressure

$\mathscr{R}_{i j} \quad$ plane-wave/plane interface reflection coefficient where wave is initially in medium $i$ and incident upon medium $j\left[=\left(\rho_{j} c_{j} / \rho_{i} c_{i}-1\right) /\right.$ $\left.\left(\rho_{j} c_{j} / \rho_{i} c_{i}+1\right)\right]$

$T_{i j} \quad$ transmission coefficient for planar interface due to plane wave initially in medium $i$ and incident upon medium $j\left[T_{i j}=2\left(\rho_{j} c_{j} / \rho_{i} c_{i}\right) /\right.$ $\left.\left(1+\left(\rho_{\mathrm{j}} \mathrm{c}_{\mathrm{j}} / \rho_{\mathrm{i}} \mathbf{c}_{\mathrm{i}}\right)\right)\right]$

1,2 subscripts indicating medium " 1 " (surrounding fluid) and medium " 2 " (body medium)

$k \quad$ acoustic wave number $(=2 \pi / \lambda)$

a radius of cylinder cross section

$P_{0} \quad$ amplitude of incident plane wave

$b_{m} \quad$ coefficient determined from boundary conditions $\phi \quad$ azimuthal angle ( $\phi=\pi$ is the backscatter direction)

$i \quad \sqrt{-1}$

$F^{(\infty)}$ form function for infinitely long cylinder

$f\left(k_{1} a\right)$ scattering amplitude for finite-sized objects (subscripts sc, ps, and bc refer to straight cylinder, prolate spheroid, and bent cylinder)

$r$ distance from axis of cylinder to the field point (applied to infinite and finite bodies)

$\mathbf{r}_{\text {pos }}$ position vector from the origin of the coordinate system to a point on the axis of the cylinder

$r_{s} \quad$ distance from a point on the axis (at $\mathbf{r}_{\text {pos }}$ ) to the field point (receiver)

$\alpha_{p} \quad$ term in divergence factor for scattered ray

$B_{p} \quad$ reflection-transmission factor

$\eta_{p} \quad$ propagation phase delay of scattered ray

$\mu_{p} \quad$ phase advance associated with crossing of caustics 
$\mu_{e} \quad$ phase shift associated with external caustics

$\epsilon_{s} \quad$ distance between the point on the axis (at $\mathbf{r}_{\mathrm{pos}}$ ) and the plane that both contains the origin and is perpendicular to the direction of the incident plane wave

$\Delta \quad k_{1} L \sin \theta$

$q \quad$ apparent volume flow per unit length of scattered field due to infinitely long cylinder

$\theta$ angle between direction of incident plane wave and plane whose normal is the tangent to the axis at each point $\mathbf{r}_{\text {pos }}$ ("angle of incidence" to local tangent)

$\hat{\mathbf{r}}_{i}, \hat{\mathbf{r}}_{r} \quad$ unit vectors describing direction of incident and received (scattered) waves

$L \quad$ length of straight finite object; arc length (or projected length, depending on context) of uniformly bent cylinder

$L_{\text {ebc }} \quad$ effective length of bent cylinder

\section{$\rho \quad$ mass density}

$\gamma \quad$ position angle of bent cylinder

$\gamma_{\max } \quad$ angle that subtends portion of bent cylinder between midpoint and end

$x \quad$ position of bent cylinder axis that has been projected onto $x$ axis

$A \quad$ (maximum deflection of bent cylinder) $/ a$

$\beta \quad$ (length of cylinder) $/ a$

$\xi \quad$ Fresnel integral parameter

$C, S \quad$ Fresnel integrals

c compressional speed of sound

g $\rho_{2} / \rho_{1}$

$h \quad c_{2} / c_{1}$

RTS target strength $-10 \log L^{2}$ (reduced target strength)

problem, simple solutions are necessary to describe average echo levels from aggregations of animals of random orientation and length.

Data collected over the past several years provide information allowing us to develop a simple model. In particular, field data collected by Pieper et al. ${ }^{10}$ and laboratory data collected by Stanton et al. ${ }^{11}$ involving average echoes from aggregations show a dip in the backscatter versus $k a$ curve toward the lower end of the geometric scattering region. Laboratory data from Chu et al. ${ }^{5}$ involving echoes from each of two individuals show a deep null in the same region. The laboratory data presented in the latter two articles showed the dip or null to consistently lie in the region surrounding $k a=2$ where $a$ is the equivalent cylindrical radius of the elongated animals (decapod shrimp whose bodies are representative of a broad class of marine organisms). The dip or null is indicative of strong interference between the acoustic wave or "ray" reflected from the front interface of the animal and a ray that has penetrated the interior with little loss and reflected off the back interface. Complementing these data are direct measurements of density and sound speed of various zooplankton that indicate that those properties are very similar (to within several percent) to those of the surrounding seawater. ${ }^{12-14}$

All of the above (direct measurement and scattering) data viewed collectively suggest that the animals tend to behave acoustically as weakly scattering bodies. For a body to be weakly scattering, the material properties must be similar to those of the surrounding medium which, as stated above, is the case for these zooplankton. Also, as will be illustrated in the theoretical and numerical portions of this article, weakly scattering bodies of certain classes of shapes (cylindrical in this case) can be described acoustically by a simple two-ray model at broadside incidence. Such a formulation gives rise to a deep null near $k a=2$ for a single target which is consistent with the data collected by Chu et $a l^{5}$ Note that even the average echo from aggregations of randomly oriented animals contains a dip 
that appears to be a "smeared" version of the null. 10,11 The dip is strong enough (of the order 5-10 dB below surrounding levels) that a model needs to be developed that predicts it.

With this significant evidence that the animals behave as weakly scattering bodies, we formulate a simple analytical approximate model that estimates the scattering levels and illustrates the fundamentals of the scattering process. Choice of this model was based, in part, on the desire for one that can be easily manipulated algebraically and is computationally efficient. These last three desirable features are not possible with the modal-series-based model described in Ref. 2 or other numerically oriented models although note that, while this current model is limited to weakly scattering materials, the modal-series-based model can be used for a broad range of materials.

The analysis is divided into two articles: this present one which involves formulating the basic physics of the scattering process for a single realization of length and angle of orientation in the region where the scattering amplitude tends to be at a maximum (near normal incidence) and a companion paper where the work is heuristically extended to include all angles of incidence for the purpose of averaging over angle. ${ }^{11}$ The resultant extended model is averaged in Ref. 11 over a range of angles and sizes to produce a model describing an "aggregation echo." The aggregation model is successfully compared in Ref. 11 with data involving aggregations of animals ranging in numbers from six to many hundreds. The data show the same 5- to 10-dB dip as predicted by the model, hence further validating the ray-based approach described in this present paper.

In this present article, a simple existing ray model is modified and incorporated in the deformed cylinder formulation. ${ }^{2}$ The modification involves heuristically altering the phase of the rays so that the ray description, which is normally valid only in the geometric or $k a \gg 1$ region, can be used accurately for values of $k a$ well below unity. While the derivation is motivated by the animal problem, the results are general and not specific to scattering by animals. Examples involving several shapes, comparison with the modal-series-based solution that involves fewer mathematical approximations, and comparison with data from single animals are given.

\section{THEORY}

\section{A. General ray-based solution}

The far-field solution to the scattering by an infinitely long cylinder is given as ${ }^{15}$

$$
p_{\text {scat }} \rightarrow P_{0} \sqrt{(a / 2 r)} e^{i k r} F^{(\infty)}, k r \gg 1,
$$

where the form function is

$$
F^{(\infty)} \equiv \frac{2}{\sqrt{\pi k a}} e^{-i \pi / 4} \sum_{m=0}^{\infty} b_{m} \cos m \phi .
$$

The modal series representation in Eq. (2) is exact for all $k a$ and material composition (axisymmetric profile). For $k a \gg 1$, however, many terms are required for mathe-
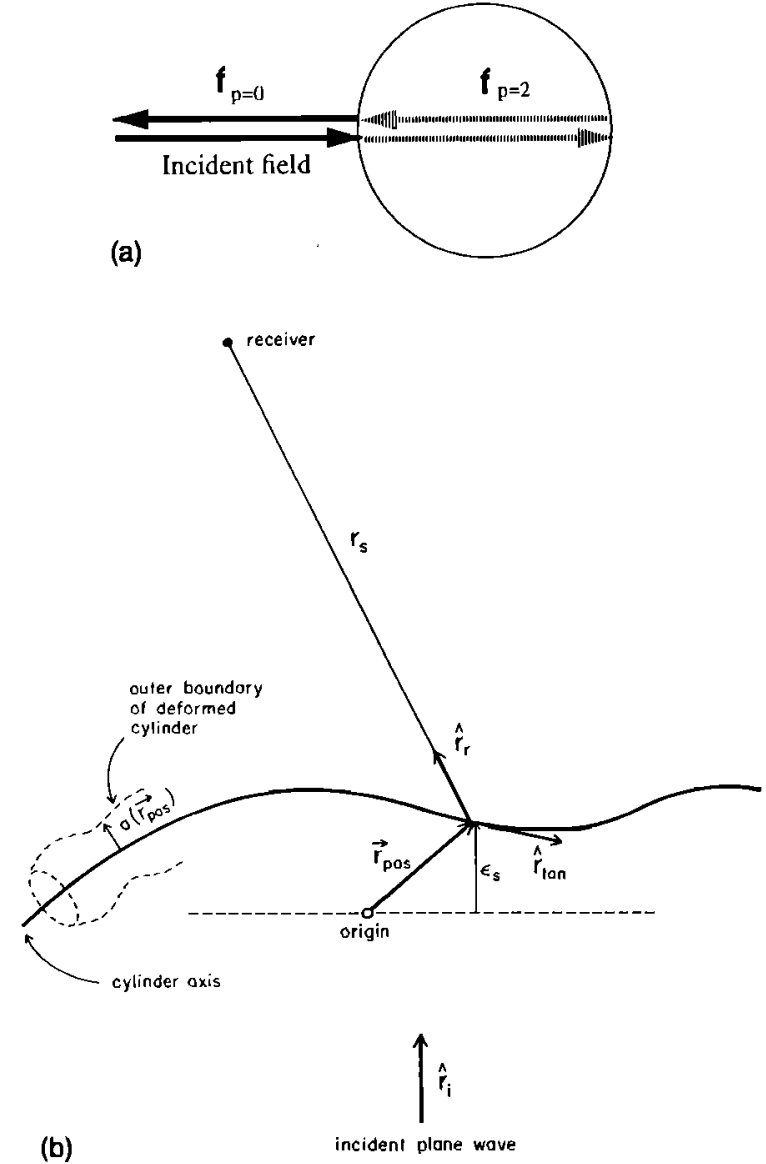

FIG. 1. (a) Weak scatterer geometry showing two types of rays scattered: "specular" echo $\left(f_{p=0}\right)$ reflected off front interface and "transmitted" echo $\left(f_{p=2}\right)$ reflected off back interface after having traveled through the body. (b) Deformed cylinder and general bistatic sonar.

matical convergence and, while modern computers can efficiently calculate the series to high precision, the series is quite cumbersome to manipulate algebraically. Use of ray solutions in this geometric scattering region has proven to facilitate the manipulations while at the same time making the physics of the scattering process more explicit in the mathematics. ${ }^{7-9,16-25}$ The ray theories are based on a variety of methods including the Sommerfeld-Watson transformation, heuristically derived formulations, and the Kirchhoff method. The success of using ray methods to describe the scattering by marine organisms is illustrated by Foote ${ }^{26}$ where the Kirchhoff method was used to numerically calculate the scattering by the front interface alone from (gas-filled) swimbladders of fish.

The types of rays that need to be taken into account depend upon the material properties. In the weak fluid scatterer case analyzed in this article $(g, h \sim 1)$, it is shown that only two rays-the ones reflected from the front and back interfaces-need to be taken into account for reasonable (although not exact) description of the scattering in the transitional and geometric region [Fig. 1(a)]. The form function associated with each ray can be calculated from the general (geometric scattering) formula for the $p$ th scattered ray from Marston: ${ }^{21}$

$$
F_{p}^{(\infty)}=(2 / a)^{1 / 2}\left|\alpha_{p}-a\right|^{1 / 2} B_{p} e^{i\left(\eta_{p}+\mu_{p}+\mu_{e}\right)},
$$


where the $p=0$ and $p=2$ rays correspond to the reflections off the front and (first-order reflection) back interfaces, respectively. The values of $p>2$ correspond to higherorder internal reflections. According to the formulas in Marston, $\quad \alpha_{p=0}=a / 2, \quad B=\mathscr{R}_{12}, \quad \eta_{p=0}=-2 k_{1} a, \quad$ and $\mu_{p=0}=0$ for the $p=0$ "specular" reflection off the front interface while $\alpha_{p=2} \simeq(3 / 2) a$ (weak scatterer approximation), $\quad B=-T_{12} T_{21} \mathscr{R}_{12}, \quad \eta_{p=2}=2 k_{1} a\left(2 h^{-1}-1\right), \quad$ and $\mu_{p=2}=-\pi / 2$ for the $p=2$ "transmitted wave" reflection off the back interface. Here, $\mu_{e}=0$ for all rays in the case presented in this article where $c_{2}>c_{1}$.

Although Eq. (3) is generally valid only in the geometric optics limit, we have found that its usefulness can be extended down to the Rayleigh/geometric transitional region $(0.1 \lesssim k a \lesssim 1)$ by heuristically removing the phase advance $\mu_{p=2}$ that is due to a caustic. This caustic is related to the curvature of the cross section of the body. Phase shifts due to the caustic naturally reduce to zero in the long wavelength limit. While the variation of phase with respect to wavelength can be derived, we remove the caustic effect gradually by replacing the constant value $\mu_{p=2}=-\pi / 2$ by the convenient function $\mu_{p=2}\left(k_{1} a\right)=-(\pi / 2) k_{1} a /$ $\left(k_{1} a+0.4\right)$. The high-frequency $\left(k_{1} a \geqslant 1\right)$ and low frequency $\left(k_{1} a<1\right)$ limits of $\mu_{p=2}\left(k_{1} a\right)$ are $-\pi / 2$ and 0 , respectively. The constant 0.4 was determined empirically and limits the errors to within about $2 \mathrm{~dB}$ for the values $k a \geqslant 0.1$ and $(g, h) \leqslant(1.1,1.1)$ for the straight cylinder.

Evaluating Eq. (3) with these parameters gives the approximate form function for the weakly scattering infinitely long fluid cylinder:

$$
\begin{aligned}
F^{(\infty)} & \simeq F_{p=0}^{(\infty)}+F_{p=2}^{(\infty)}, \\
& =\mathscr{R}_{12} e^{-i 2 k_{1} a}\left(1-T_{12} T_{21} e^{i 4 k_{2} a} e^{i \mu_{p=2}\left(k_{1} a\right)}\right),
\end{aligned}
$$

where the general form for the scattering is given in Eq. (4) and an explicit approximate form is in Eq. (5). The assumptions for these equations are (1) the incident plane wave is traveling in a direction normal to the axis of the cylinder, (2) the cylinder is surrounded by a fluid, and (3) the scattering is sufficiently weak that (a) the first reflection off the back interface dominates the higher-order internal reflections and (b) bending of the rays at the interface (Snell's law) is negligible. Simulations later in this article verify the validity of assumption (3).

A useful property of the scattered field from elongated, deformed, sometimes finite bodies is the apparent volume flow per unit length $q$ of the field. ${ }^{1,2}$ This term can be integrated over the length of the body to provide estimates of the scattered field, and hence backscattering cross section and target strength. The accuracy of the estimates improve as the aspect ratio (length/diameter) increases. The volume flow per unit length will be used in predicting scattering by the finite objects in this article.

The far-field-scattered pressure due to a plane-wave incident upon an infinitely long cylinder is written in terms of $q$ as

$$
p_{\text {scat }} \rightarrow\left(e^{i k_{1} r} / \sqrt{r}\right) q e^{-i \pi / 4} \sqrt{k_{1}} \rho_{1} c_{1} /(2 \sqrt{2 \pi}) \quad\left(k_{1} r \gg 1\right),
$$

where

$$
q=\frac{4 P_{0}}{k_{1} \rho_{1} c_{1}} \sum_{m=0}^{\infty} b_{m} \cos m \phi .
$$

The volume flow per unit length can be related to the form function with the following expression:

$$
q=2 P_{0} \sqrt{\left(\pi a / k_{1}\right)} e^{i \pi / 4} F^{(\infty)} / \rho_{1} c_{1} .
$$

Inserting this equation in Eqs. (4) and (5) gives an approximate expression for the volume flow per unit length due to the scattering from a weakly scattering fluid cylinder:

$$
\begin{aligned}
q \simeq & q_{p=0}+q_{p=2}, \\
= & 2 P_{0} \mathscr{R}_{12} e^{-i 2 k_{1} a}\left(1-T_{12} T_{21} e^{i 4 k_{2} a} e^{i \mu_{p=2}\left(k_{1} a\right)}\right) \\
& \times e^{i \pi / 4} \sqrt{\pi a} /\left(\rho_{1} c_{1} \sqrt{k_{1}}\right) .
\end{aligned}
$$

The above volume flow term is incorporated into the deformed cylinder formulation so that effects due to deformities such as bend, taper, and roughness can be estimated. The approximate formulation allows for calculation of finite or infinitely long bodies. The general solution for bodies of any length is given as

$$
\begin{aligned}
p_{\text {scat }}= & \frac{-i}{4 \pi} \rho_{1} c_{1} k_{1} \int_{\mathrm{r}_{\mathrm{pos}}} q \cos \theta \frac{e^{i k_{1}\left(r_{s}+\epsilon_{s}\right)}}{r_{s}}\left|d \mathrm{r}_{\mathrm{pos}}\right| \\
& \times \text { (any length), }
\end{aligned}
$$

where some of the terms are illustrated in Fig. 1(b). All wavenumbers in $q$ are modified by the factor $\cos \theta$. Note that in Eq. (4) of Ref. 2 there is no explicit $\cos \theta$ term that corresponds to the one that appears in the above expression. The misleading omission in Ref. 2 made no difference in the results because from Eq. (5) and beyond, the modal series representation of $q$ was used and a $(\cos \theta)^{-1}$ term in $q$ cancelled out the would-be $\cos \theta$ factor. In the ray representation, there is no such cancellation hence the $\cos \theta$ is written explicitly. The solution for bodies of finite length (i.e., the length is much less than the first Fresnel zone of the source/receiver combination) simplifies to

$$
p_{\text {scat }}=P_{0}\left(e^{i k_{1} r} / r\right) f\left(k_{1} a\right),
$$

where

$$
\begin{aligned}
f\left(k_{1} a\right) \simeq & \left(P_{0}\right)^{-1}\left(\frac{-i k_{1} \rho_{1} c_{1}}{4 \pi}\right) \int_{\mathrm{r}_{\mathrm{pos}}} q \cos \theta \\
& \times \exp \left[i k_{1} r_{\mathrm{pos}}\left(\hat{r}_{i}-\hat{r}_{r}\right) \cdot \hat{r}_{\mathrm{pos}}\right]\left|d \mathbf{r}_{\mathrm{pos}}\right| \\
& \text { (finite length). }
\end{aligned}
$$

The details of these equations are discussed in Refs. 2 and 8. Note that the implicit $P_{0}$ in $q$ cancels the explicit $\left(P_{0}\right)^{-1}$ in Eq. (12b).

\section{B. Deformed smooth bodies}

The ray formulation can readily be incorporated into the above deformed cylinder equations by direct substitution to obtain approximate solutions to the scattering by a 
variety of bodies such as the straight finite cylinder, prolate spheroid (high aspect ratio), and uniformly bent cylinder. Adaptation of the integral formulation to each shape is described in Refs. 2 and 8 and includes (1) allowing the "cylindrical radius" of the prolate spheroid to vary along the lengthwise axis, (2) taking into account the phase shifts induced by the bend of the axis of the bent cylinder (this takes place in the exponent in the integrand), and (3) solving the integral along the length of the cylinders for the prolate spheroid and bent cylinder by use of the method of stationary phase or Fresnel integrals. Performing the above manipulations gives the following approximate solutions for those shapes in the geometric scattering region:

1. Straight cylinder (various angles of incidence):

$$
\begin{aligned}
f_{\mathrm{sc}}= & \frac{-i}{2 \sqrt{\pi}} e^{i \pi / 4} e^{-i 2 k_{1} a \cos \theta} L \sqrt{k_{1} a \cos \theta} \mathscr{R}_{12} \frac{\sin \Delta}{\Delta} \\
& \times\left(1-T_{12} T_{21} e^{i 4 k_{2} a \cos \theta} e^{i \mu_{p=2}\left(k_{1} a\right)}\right) .
\end{aligned}
$$

2. Prolate spheroid (broadside incidence):

$$
f_{\mathrm{ps}}=(1 / 4) L \mathscr{R}_{12} e^{-i 2 k_{1} a_{0}}\left(1+i T_{12} T_{21} e^{i 4 k_{2} a_{0}} e^{i \mu_{p=2}\left(k_{1} a\right)}\right) .
$$

3. Bent cylinder (uniformly bent or nearly so; bent symmetrically away from sonar).

a. Arbitrary deflections. For values of deflection of the bent cylinder that are arbitrary with respect to wavelengths of sound, one obtains

$$
\begin{aligned}
f_{\mathrm{bc}} \simeq & \frac{-i}{2 \sqrt{\pi}} \sqrt{k_{1} a} L_{\mathrm{ebc}} \mathscr{R}_{12} e^{-i 2 k_{1} a}(1 \\
& \left.-T_{12} T_{21} e^{i 4 k_{2} a} e^{i \mu_{p=0}\left(k_{1} a\right)}\right) e^{i \pi / 4}, \\
= & L_{\mathrm{ebc}} f_{\mathrm{sc}}\left(\theta=0^{\circ}\right) / L,
\end{aligned}
$$

where the approximations $k_{2}=k_{1}$ and $\cos \gamma \sim 1$ were made in amplitude terms and the limit $\rho_{c} \gg a$ was used (which is reasonable for elongated zooplankton). The effective length $L_{\text {ebc }}$ can be expressed in terms of either the radius of curvature $\rho_{c}$ of the cylinder axis or deflection $z(x)=4 A x^{2} / \beta^{2} a$ of the axis from a straight line:

$$
L_{\mathrm{ebc}}=\rho_{c} \int_{-\gamma_{\max }}^{\gamma_{\max }} e^{i k_{1} \rho_{c} \gamma^{2}} d \gamma \quad(\gamma \ll 1)
$$

and

$$
L_{\mathrm{ebc}}=\int_{-L / 2}^{L / 2} e^{i 8 k_{1} A x^{2} / \beta^{2} a} d x
$$

The term $\gamma$ in Eq. (16a) denotes the position angle on the uniformly bent cylinder and $2 \rho_{c} \gamma_{\max }=L$ (see Ref. 2 for geometry). The terms chosen in the exponent in Eq. (16b) relate the curvature of the axis to a parabolic curve. Here, $z(L / 2)=A a, L=\beta a$, and $x$ is the position along the cylinder projected onto a straight line (see Ref. 7 for geometry). $\beta$ and $A$ are parameters indicating the length and deflection in terms of the cylindrical radius. These equations have the form of Fresnel integrals. As was done in Ref. 7, a change in the variables to the following parameters is performed for Eqs. (16a) and (16b), respectively:

$$
(\pi / 2) \xi^{2} \equiv k_{1} \rho_{c} \gamma^{2} \text { and } \xi_{1} \equiv \sqrt{(2 / \pi) k_{1} \rho_{c}} \gamma_{\max }
$$

and

$$
(\pi / 2) \xi^{2}=8 k_{1} A x^{2} / \beta^{2} a \text { and } \xi_{1} \equiv 2 \sqrt{k_{1} A a / \pi},
$$

which results in the compact formulas:

$$
L_{\mathrm{ebc}}=\sqrt{\rho_{c} \lambda_{1}}\left[C\left(\xi_{1}\right)+i S\left(\xi_{1}\right)\right]
$$

and

$$
L_{\mathrm{ebc}}=L \sqrt{\lambda_{1} / 8 A a}\left[C\left(\xi_{1}\right)+i S\left(\xi_{1}\right)\right],
$$

for Eqs. (16a) and (16b), respectively, where $A a$ is the maximum deflection of the cylinder, $\sqrt{\rho_{c} \lambda_{1} / 2}$ is the radius of the first Fresnel zone of the bent cylinder, and the Fresnel integrals $C$ and $S$ are given as

$$
\begin{aligned}
& C\left(\xi_{1}\right)=\int_{0}^{\xi_{1}} \cos \left(\frac{\pi}{2} \xi^{2}\right) d \xi \text { and } \\
& S\left(\xi_{1}\right)=\int_{0}^{\xi_{1}} \sin \left(\frac{\pi}{2} \xi^{2}\right) d \xi .
\end{aligned}
$$

The Appendix contains expressions for $C$ and $S$ for arbitrary bends (i.e., deflections are not restricted with respect to wavelength).

b. Small deflections. In the limit of deflections of the bent cylinder that are small compared with a wavelength, the above equations reduce to the straight cylinder case and $L_{\mathrm{ebc}}=L$.

c. Large deflections. For deflections of the bent cylinder much greater than a certain fraction of a wavelength, or more precisely, $k_{1} \rho_{c} \gamma_{\max }^{2}>1$ or $8 k_{1} A L^{2} / \beta^{2} a \gg 1$, one can use the method of stationary phase or asymptotic limits of the Fresnel integrals (the Appendix) to solve for $L_{\mathrm{ebc}}$ in Eqs. (16a) and (16b):

$$
L_{\mathrm{ebc}} \simeq \sqrt{\rho_{c} \lambda_{1} / 2} e^{i \pi / 4}
$$

and

$$
L_{\mathrm{ebc}} \simeq(L / 4) \sqrt{\lambda_{1} / A a} e^{i \pi / 4},
$$

respectively. Substituting these expressions into Eq. (15b) gives

$$
\begin{aligned}
& f_{\mathrm{bc}}=\sqrt{\rho_{c} \lambda_{1} / 2} e^{i \pi / 4} f_{\mathrm{sc}}\left(\theta=0^{\circ}\right) / L, \\
& f_{\mathrm{bc}}=\frac{1}{4} \sqrt{\lambda_{1} / A a} e^{i \pi / 4} f_{\mathrm{sc}}\left(\theta=0^{\circ}\right),
\end{aligned}
$$

where the scattering amplitude is shown to be equal to within a phase factor to the product of the radius of the first Fresnel zone of the bent cylinder and the normalized scattering amplitude of the straight cylinder at normal incidence in Eq. (21a) and proportional to the product of the square root of the ratio of the wavelength to maximum deflection of the bent cylinder and straight cylinder scattering amplitude in Eq. (21b).

\section{NUMERICAL SIMULATIONS}

In this section, the ray solution is explored under a variety of conditions. The material properties and shape 


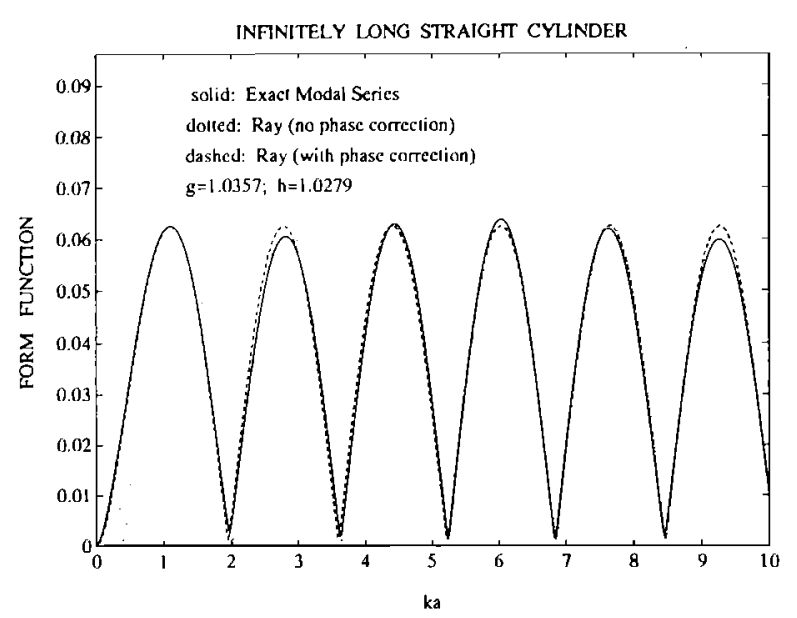

FIG. 2. Comparison between simple approximate ray solution [Eq. (5)] illustrated in Fig. 1, and the exact modal-series solution [Eq. (2), mathematically converged] for weakly scattering infinitely long smooth fluid cylinder $\left(k \equiv k_{1}\right)$. Equation ( 5 ) is evaluated for both $\mu_{\rho=2}=-\pi / 2$ (no phase correction) and $\mu_{p=2}=-(\pi / 2) k_{1} a /\left(k_{1} a+0.4\right)$ (phase correction) where in the latter case, the phase shift due to the caustic is heuristically removed in the low $k a$ region. The ray solution is remarkably similar to the exact solution for small contrast in material properties $[(g, h) \lesssim(1.01,1.01)$, not shown], especially once the caustic-related phase shift is removed for small $k a$. For the zooplankton material properties used in the example shown, the accuracy is quite satisfactory. Similar accuracies were observed for $k a>10$.

are varied and compared with the modal-series-based solution to illustrate the usefulness and range of validity of the approximate solution. All calculations involve backscattering.

The weak scatterer approximation is tested in Fig. 2 where the ray solution is compared with the exact modal series solution for values of speed of sound and density similar to those observed for marine organisms. ${ }^{14}$ The ray solution is given for cases with and without the phase correction. There are significant differences between the ray solution without the correction and the exact solution while, once corrected, the ray solution follows the exact solution to a fair degree of accuracy. For values of $g$ and $h$ greater than 1.1, the ray model no longer is valid as the peaks in the curve will deviate by more than $10 \%$. Similar to the analysis of Clay, ${ }^{7}$ the impulse response ${ }^{15,27}$ of the form function of the exact solution from Fig. 2 is shown in Fig. 3 to decompose the echo into the different arrivals caused by an incident impulsive (delta function) signal. The time of arrival of the two echoes observed correspond to reflections from the front and back interfaces of the cylinder. The ray approach is incorporated into the deformed cylinder formulation in Fig. 4 to estimate the scattering by finite length bodies. Both the modal-series-basedand ray-based solutions are illustrated for comparison. Note that although the modal series solution is exact for the smooth infinitely long cylinder, the deformed cylinder solution is approximate, hence both sets of curves shown in Fig. 4 involve different degrees of approximation. Details of the calculations involved in Fig. 4 are discussed in Ref. 2.

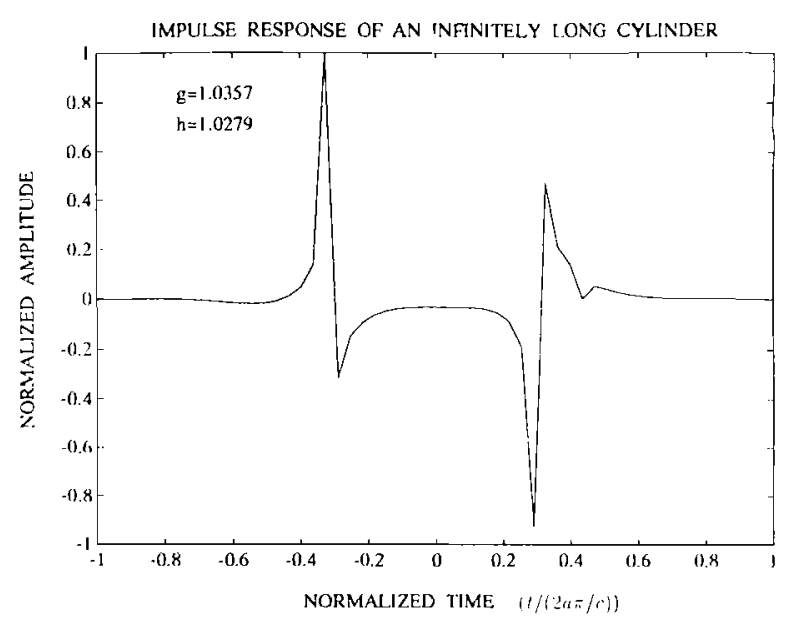

FIG. 3. Impulse response of exact (modal series) solution to infinitely long weakly scattering fluid cylinder (solution taken from Fig. 2 with the high $k a$ values tapered via the edge of a Hanning window). The first $\left(f_{p=0}\right)$ and second $\left(f_{p=2}\right)$ arrivals are illustrated.

\section{COMPARISON WITH LABORATORY DATA}

In a recent study published by Chu et al., the backscatter data from two live decapod shrimp were presented and analyzed. ${ }^{5}$ The animals were tethered by human hair and individually insonified over the frequency range $300-$ $650 \mathrm{kHz}$ at near-normal incidence. In order to mount the animals, they were anesthetized, although note that they changed shape and orientation throughout the experiment (i.e., the medication slowed them down, but did not render them lifeless). Because of the motion of the animals, the echo level and spectral shape subsequently changed in time. To study this effect, many echoes were recorded from each animal representing a range of realizations of shape and orientation. The resultant plots of backscatter versus $k a$ indicated strong modal resonances with nulls ranging in level from 10 to $30 \mathrm{~dB}$ below the surrounding value. The position of the nulls tended to shift slightly from realization to realization as one would expect for a body that is undergoing changes in shape and orientation. Once all of the echoes were averaged (incoherently) in the computer, the nulls were mostly "washed out" leaving more gradual dips of the order 5-8 dB below local levels.

One plot representative of the single echoes or realizations was compared with a single realization of the modalseries-based bent cylinder solution. There was reasonable qualitative comparison between the structure of the data and theory in that the general $(k a)$ position of the peaks and nulls generally agreed. There was no basis for comparison between the absolute levels as the precise shape and orientation of the animal were not known.

The modal-series-based solution is replaced in Fig. 5 with the simpler, ray-based model. A similar degree of qualitative correlation between the ray model and data exists as with the modal solution (see above discussion). As in the analysis involving the modal-series-based solution, it is important to point out that the comparison with the ray-based model should be qualitative also since the precise 


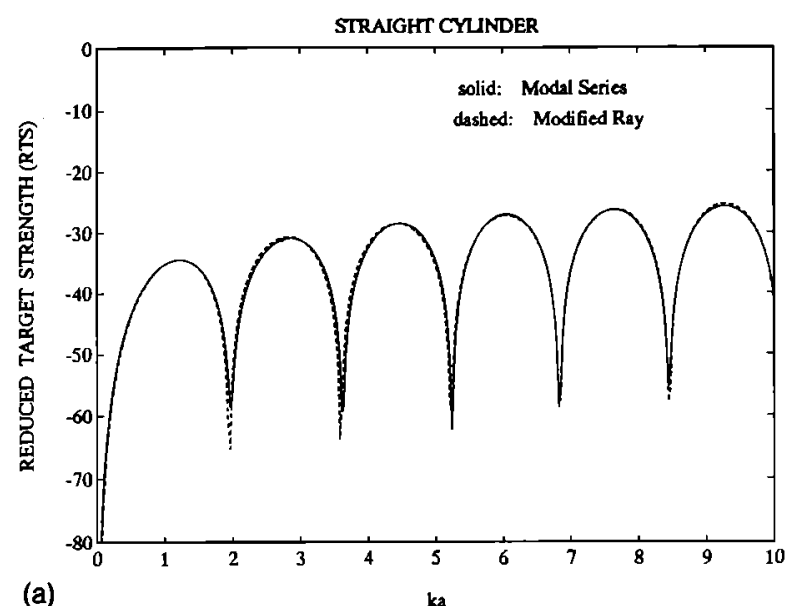

(a)

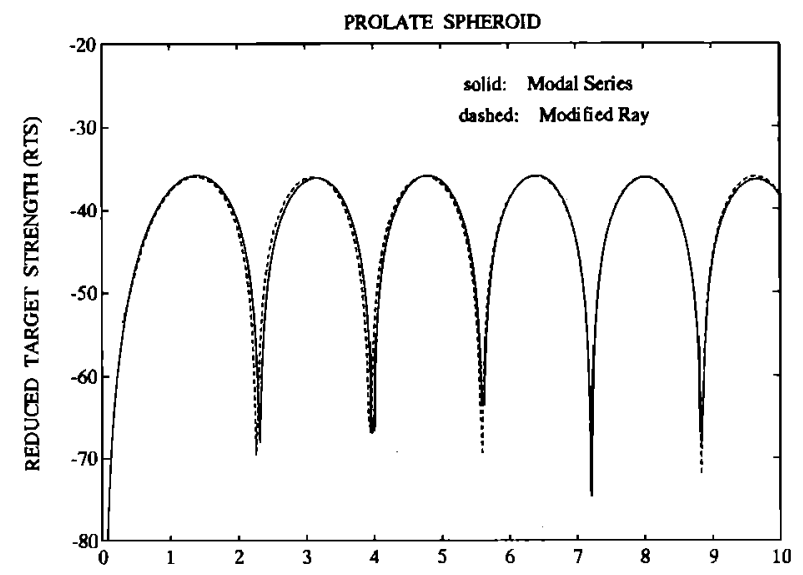

(b)

ka

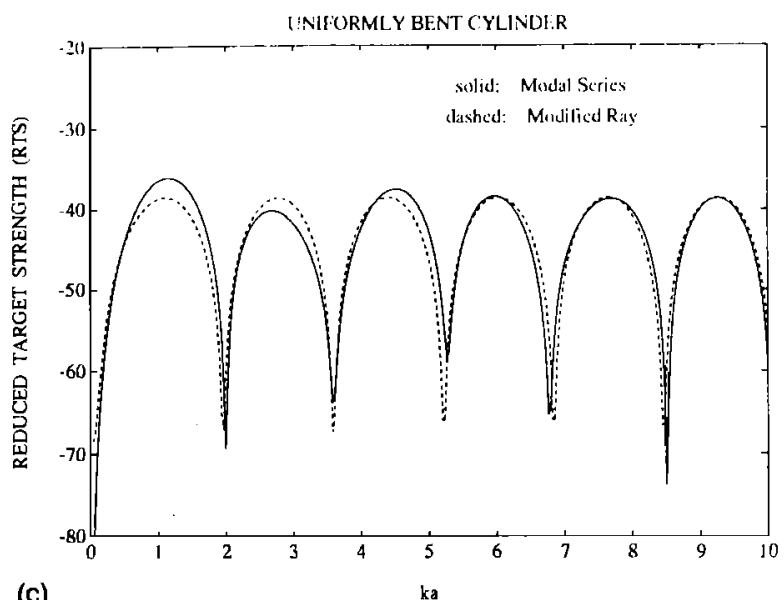

(c)

FIG. 4. Ray and modal series solutions, each incorporated into deformed cylinder solution, to estimate scattering by various deformed elongated finite length smooth bodies. The following (zooplanktonlike) parameters were used in the calculations: $(g, h)=(1.0357,1.0279)$ (all plots), and $\rho_{d} / L=1.5$ and $L / a=10.5$ (bent cylinder). The prolate spheroid calculations are valid for $L / 2 a \gtrsim 5$. The modal series is mathematically converged in each plot. Equations (13), (14), and (21a) were used in the (phase corrected or "modified") ray calculations while Eqs. (7) and (12b) were used for the modal-series curves. Similar accuracies were observed for $k a>10$.

shape and orientation of the animals were not known. It is only the trend and general positions of peaks and nulls of the data and model that should be compared, not absolute level and precise positions.

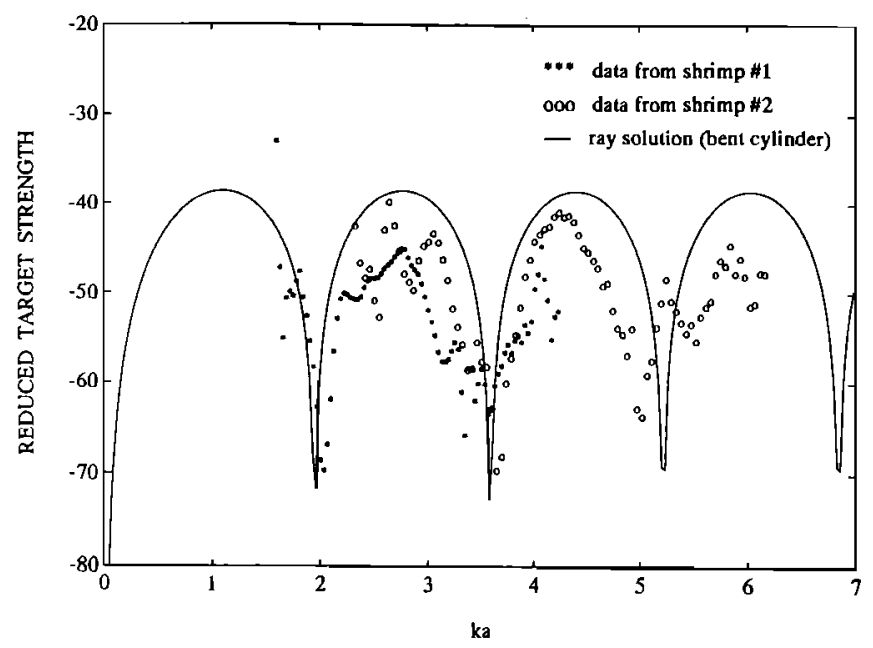

FIG. 5. Comparison between shrimp backscatter data (near normal incidence) collected by Chu et $a l^{5}$ and (phase corrected) ray-based bent cylinder model [Eq. (2la)]. Same parameters are used in this model as those used in modal-series-based modeling in Chu et al. ( $\rho_{\mathrm{c}} L=1.5, L / a$ $=10.5$, and $g, h=1.0357,1.0279) .{ }^{5}$ Here, $a$ is the average cylindrical radius of the thorax section of each shrimp. The simple model predicts the general locations of nulls as well as overall levels although note that the change of shape and orientation of the live moving animals caused the locations and levels to shift from echo to echo (not shown).

\section{DISCUSSION AND CONCLUSIONS}

Perhaps one of the most notable results of this article is the simplicity of the (approximate) two-ray solution and the close agreement between it and the exact modal series solution. Equation (5) shows that the ray solution for weakly scattering infinitely long straight cylinders is the summation of two simple terms which is in sharp contrast with the much more complex (exact) modal series solution given in Eq. (2). The simulations show that for weakly scattering fluid cylinders, the ray and series solutions are quite close to each other. In fact, as $g$ and $h$ are reduced to near unity, the ray solution becomes indistinguishable from the modal series solution over a wide range of $k a$ (not shown). In addition, the real and imaginary components of each solution (not shown) compare very well with the corresponding component of the other solution. Once the density and speed of sound contrasts are increased the ray and series solutions begin to diverge [a discrepancy that is not corrected by removing the weak scatterer approximation in $\alpha_{p}$ in the divergence factor in Eq. (3)]. Certainly other phenomena such as including more rays would improve the accuracy of those results. ${ }^{21}$ The fact that the scattering by weakly scattering cylinders was dominated by the rays reflected from the front and back interfaces was illustrated in Fig. 3 when the impulse response of the modal series solution was calculated. The resultant echo was composed of two separate signals whose times of arrival were consistent with what one would expect for the two rays. These times compared exactly with those of $\mathrm{Clay}^{7}$ who performed a similar calculation. When the ray solution was incorporated into the deformed cylinder so- 
lution for the deformed objects in Fig. 4, the ray-based solution compared well with the modal-series-based solution.

Another interesting feature of the plots is the fact that, although ray solutions typically are accurate only for very high $k a$, the "phase corrected" one illustrated in Fig. 2 appears to maintain reasonable accuracy down to about $k a=0.1$. In particular, the phase-corrected ray-based deformed cylinder solution was accurate down to values of $k a=0.1,0.02$, and 0.3 for the straight finite cylinder, bent cylinder, and prolate spheroid, respectively. As discussed in Sec. I A, the correction involves heuristically removing the phase shift induced by a (high-frequency region) caustic in the low-frequency region. Accuracy in this lowfrequency region is essential in using sonars to classify zooplankton.

The usefulness of the ray-based deformed cylinder solution was illustrated in Fig. 5 where it was compared with the data set from Chu et al. The simple solution compared reasonably well on a qualitative basis with the trend and structure of the scatter pattern. Certainly it compared to the same degree as the approximate modal-series-based solution did in Ref. 5. While this plot involved only one realization of animal shape, the data from other echoes (some of which are shown in Ref. 5) contained similar structure. In particular, all data contained peaks and nulls (or dips) occurring in the same general location as that predicted by the single realization predictions. The shifting of their locations from echo to echo is undoubtedly due to the constantly changing shape and orientation of the animals. A more rigorous analysis in predicting the precise locations of the peaks and nulls and absolute levels would require knowledge of the precise shape and orientation of the animals. Nonetheless, the favorable comparison suggests that the animals (decapod shrimp) can be treated as weakly scattering fluid bodies whose scattering properties near-normal incidence can be approximated by a simple two-ray model.

In addition to the major peaks and nulls mentioned above, there also appear to be some smaller ones occurring near $k a=2.3,3.2,4.2$ (possibly), and 5.4. Since the structure is so weak and the data set so limited, explanation regarding the mechanism causing the oscillations would be purely speculative. However, their near-unity periodicity does, in fact, correspond to the periodicity one would expect due to interferences between circumferential waves traveling near the speed of sound of the zooplankton and surrounding water. Whether of not such waves exist with these animals remains to be seen.

In conclusion, the simple two-ray-based deformedcylinder solution has proven to be a good descriptor of the scattering by idealized weakly scattering fluid objects nearnormal incidence. The model, besides having the advantage of illustrating the basic physics of the scattering, is in such a form that can be manipulated analytically and is easy to compute. The reasonable qualitative comparison of the structure of the ray solution with the shrimp data suggests that the irregularly shaped animals can be modeled as weak fluid scatterers whose scattering properties can be approximated, at least near-normal incidence, by a simple two-ray model. Although the scattering structure predicted by the single realization ray model is very sensitive to the precise shape and orientation of the animals, the sensitivity is dramatically reduced once ensemble averages with respect to length and orientation of the extended solution are performed in Ref. 11. In that companion article, the deep nulls from the single realization solution become rounded "dips" due to the averaging. The averages compare very well with data involving aggregations of up to hundreds of animals. Hence, the end result in that analysis involving many animals further validates use of the raybased formulas to describe scattering in the single realization case.

\section{ACKNOWLEDGMENTS}

The authors are grateful to the following people from the Woods Hole Oceanographic Institution, Woods Hole, MA: Bob Eastwood for his aid in the numerical computations on the computer and Shirley Bowman for preparing the manuscript to this article. This work was supported by the U.S. Office of Naval Research Grant No. N00014-89J-1729 and National Science Foundation Grant No. OCE8817171. This is contribution numbers 8029 and 527 for the Woods Hole Oceanographic Institution and Geophysical and Polar Research Center, University of WisconsinMadison, respectively.

\section{APPENDIX: CONVENIENT FORMULAS FOR FRESNEL INTEGRALS}

Numerical expressions for the evaluation of Fresnel integrals are in Abramowitz and Stegun, Sec. 7.3. ${ }^{28} \mathrm{We}$ repeat the expressions here for the readers' convenience. The notation for the real variable $x$ is not related to $x$ used in the main text. The equation numbers A\&S are those in the handbook.

$$
C(x)=\int_{0}^{x} \cos \left(\frac{\pi}{2} t^{2}\right) d t
$$

and

$$
S(x)=\int_{0}^{x} \sin \left(\frac{\pi}{2} t^{2}\right) d t
$$

For real variables and using the auxiliary functions $f(x)$ and $g(x)$, the Fresnel integrals are

$$
\begin{aligned}
& C(x)=\frac{1}{2}+f(x) \sin \left(\frac{\pi}{2} x^{2}\right)-g(x) \cos \left(\frac{\pi}{2} x^{2}\right), \\
& S(x)=\frac{1}{2}-f(x) \cos \left(\frac{\pi}{2} x^{2}\right)-g(x) \sin \left(\frac{\pi}{2} x^{2}\right)
\end{aligned}
$$

Rational approximations for the auxiliary functions $(0 \leqslant x \leqslant \infty)$ are

$$
f(x)=\frac{1+0.926 x}{2+1.792 x+3.104 x^{2}}+\epsilon(x), \quad|\epsilon(x)| \leqslant 0.002,
$$




$$
\begin{aligned}
g(x)= & \frac{1}{2+4.142 x+3.492 x^{2}+6.670 x^{3}}+\epsilon(x), \\
& |\epsilon(x)| \leqslant 0.002 .
\end{aligned}
$$

${ }^{1}$ T. K. Stanton, "Sound scattering by cylinders of finite length. I. Fluid cylinders," J. Acoust. Soc. Am. 83, 55-63 (1988).

${ }^{2}$ T. K. Stanton, "Sound scattering by cylinders of finite length. III. Deformed cylinders," J. Acoust. Soc. Am. 86, 691-705 (1989).

${ }^{3}$ P. H. Wiebe, C. H. Greene, T. K. Stanton, and J. Burczynski, "Sound scattering by live zooplankton and micronekton: Empirical studies with a dual-beam acoustical system," J. Acoust. Soc. Am. 88, 23462360 (1990)

${ }^{4}$ C. H. Greene, T. K. Stanton, P. H. Wiebe, and S. McClatchie, “Acoustic estimates of Antarctic krill," Nature, 349, 110 (1991).

${ }^{5}$ D. Chu, T. K. Stanton, and P. H. Wiebe, "On the frequency dependence of sound backscattering from live zooplankton," ICES J. Mar. Sci. 49, 97-106 (1992).

${ }^{6}$ K. G. Foote, D. Chu, and T. K. Stanton, "Status of krill target strength," CCAMLR Selected Scientific Papers (in press).

${ }^{7}$ C. S. Clay, "Low-resolution acoustic scattering models: Fluid-filled cylinders and fish with swimbladders," J. Acoust. Soc. Am. 89, 2168-2179 (1991).

${ }^{8}$ T. K. Stanton, "Sound scattering by rough elongated elastic objects: I. Means of scattered field," J. Acoust. Soc. Am. 92, 1641-1664 (1992).

${ }^{9}$ T. K. Stanton and D. Chu, "Sound scattering by rough elongated elastic objects: II. Fluctuations of scattered field," J. Acoust. Soc. Am. 92, 1665-1678 (1992).

${ }^{10}$ R. E. Pieper, D. V. Holliday, and G. S. Kleppel, "Quantitative zooplankton distributions from multifrequency acoustics," J. Plankton Res. 12, 433-441 (1990).

"T. K. Stanton, D. Chu, P. H. Wiebe, and C. S. Clay, "Average echoes from randomly oriented random-length finite cylinders: Zooplankton models," J. Acoust. Soc. Am. 94, 3463-3472 (1993).

${ }^{12}$ C. F. Greenlaw, "Backscattering spectra of preserved zooplankton," J. Acoust. Soc. Am. 62, 44-52 (1977).

${ }^{13}$ K. G. Foote, I. Everson, J. L. Watkins, and D. G. Bone, "Target strengths of Antarctic krill (Euphausia superba) at 38 and $120 \mathrm{kHz}$," J. Acoust. Soc. Am. 87, 16-24 (1990).
${ }^{14}$ K. G. Foote, "Speed of sound in Euphausia superba," J. Acoust. Soc. Am. 87, 1405-1408 (1990).

${ }^{15}$ E. Skudrzyk, The Foundations of Acoustics (Springer-Verlag, New York, 1971)

${ }^{16} \mathrm{E}$. Heyman and L. Felsen, "Creeping waves and resonances in transient scattering by smooth convex objects," IEEE Trans. Ant. Prop. AP-31, 426-437 (1983)

${ }^{17}$ L. B. Felsen, J. M. Ho, and I. T. Lu, "Three-dimensional Green's function for fluid-loaded thin elastic cylindrical shell: Formulation and solution," J. Acoust. Soc. Am. 87, 543-553 (1990).

${ }^{18}$ L. B. Felsen, J. M. Ho, and I. T. Lu, "Three-dimensional Green's function for fluid-loaded thin elastic cylindrical shell: Alternative representations and ray acoustic forms," J. Acoust. Soc. Am. 87, 554-569 (1990).

${ }^{19}$ N. D. Veksler and V. M. Korsunskii, "On the acoustic pressure formula for backscattering by thin elastic circular cylindrical shells," Acoust. Lett. 6, 70-78 (1982).

${ }^{20}$ P. L. Marston, "GTD for backscattering from elastic spheres and cylinders in water and the coupling of surface elastic waves with the acoustic field," J. Acoust. Soc. Am. 83, 25-37 (1988).

${ }^{21}$ P. L. Marston, "Geometrical and catastrophe optics methods in scattering," in Physical Acoustics, edited by A. D. Pierce and R. N. Thurston (Academic, New York, 1992), Vol. 21.

${ }^{22} \mathrm{H}$. Überall, "Surface waves in acoustics," in Physical Acoustics, edited by W. P. Mason and R. N. Thurston (Academic, New York, 1973), Vol. 10, pp. 1-60.

${ }^{23} \mathrm{G}$. C. Gaunaurd, "Sonar cross sections of bodies partially insonified by finite sound beams," IEEE J. Ocean. Eng. OE-10, 213-230 (1985).

${ }^{24} \mathrm{H}$. M. Nussenzveig, "High frequency scattering by an impenetrable sphere," Ann. Phys. 34, 23-95 (1965).

${ }^{25}$ H. M. Nussenzveig, "High frequency scattering by a transparent sphere. I. Direct reflection and transmission," J. Math. Phys. 10, 82124 (1969).

${ }^{26} \mathrm{~K}$. G. Foote, "Rather-high-frequency sound scattering by swimbladdered fish," J. Acoust. Soc. Am. 78, 688-700 (1985).

${ }^{27}$ Z. Sun, G. Gimenez, D. Vray, and F. Dedis, "Calculation of the impulse response of a rigid sphere using the physical optic method and modal method jointly," J. Acoust. Soc. Am. 89, 10-18 (1991).

${ }^{28} \mathrm{M}$. Abramowitz and I. Stegun, Handbook of Mathematical Functions (U.S. Government Printing Office, Washington, DC, 1964). 